\title{
Corela
}

Cognition, représentation, langage

HS-18 | 2015

La reformulation : usages et contextes

\section{La reformulation et multimodalité dans un cours en visioconférence}

Pascale TRÉVISIOL-OKAMURA et Martine MARQUILLÓ-LARRUY

\section{(2) OpenEdition}

\section{Journals}

\section{Édition électronique}

URL : http://journals.openedition.org/corela/4155

DOI : $10.4000 /$ corela.4155

ISSN : $1638-573 \mathrm{X}$

\section{Éditeur}

Cercle linguistique du Centre et de I'Ouest - CerLICO

\section{Édition imprimée}

Date de publication : 15 novembre 2015

ISSN : 1638-5748

\section{Référence électronique}

Pascale TRÉVISIOL-OKAMURA et Martine MARQUILLÓ-LARRUY, « La reformulation et multimodalité dans un cours en visioconférence », Corela [En ligne], HS-18 | 2015, mis en ligne le 15 novembre 2015 consulté le 01 mai 2019. URL : http://journals.openedition.org/corela/4155 ; DOI : 10.4000/ corela.4155

Ce document a été généré automatiquement le 1 mai 2019.

\section{(c) (i) (2)}

Corela - cognition, représentation, langage est mis à disposition selon les termes de la licence Creative Commons Attribution - Pas d'Utilisation Commerciale - Partage dans les Mêmes Conditions 4.0 International. 


\title{
La reformulation et multimodalité dans un cours en visioconférence
}

\author{
Pascale TRÉVISIOL-OKAMURA et Martine MARQUILLÓ-LARRUY
}

\section{Introduction}

1 L'apprentissage d'une langue par "visio-conférence poste à poste" fait partie de ces nouveaux dispositifs qui ouvrent des voies encore peu balisées vers un apprentissage renouvelé des langues. Le dispositif Le français en (première) ligne, initialement imaginé et développé par Christine Develotte et ses collègues ${ }^{1}$ est expérimenté depuis l'année universitaire 2011-2012 dans le cadre du master professionnel de didactique des langues et du français langue étrangère et seconde (Didalang-FLE/S) de l'université de Poitiers ${ }^{2}$ et des cours de français langue étrangère dispensés à Télécom École de Management et Télécom SudParis (école d'ingénieurs) à Evry sous la responsabilité de Laure Chotel. Ce dispositif, qui met en relation des étudiants-tuteurs francophones et des apprenants allophones distants, permet de former aux régulations pédagogiques (cf. Guichon \& Drissi, 2008) et à l'élaboration d'outils (tâches multimédia), tout en suscitant des échanges en ligne autour de ces tâches avec de "vrais" apprenants.

2 Cette contribution explore et cherche à décrire quelques modalités de reformulation spontanées des tuteurs ${ }^{3}$ face aux réactions des apprenants. La spontanéité est ici associée au fait que les étudiants n'ont pas reçu une formation spécifique pour travailler la reformulation. La conversation en ligne inscrit la communication dans un contexte de médiation technologique. Bien que les pratiques interactionnelles soient influencées par les aspects techniques de ce dispositif (nous en montrerons un exemple), nous mettrons davantage l'accent sur le répertoire de stratégies reformulatives et explicatives observables dans notre corpus multimodal. Comme le souligne Alain Rabatel (2010:7), en introduction à l'ouvrage Les reformulations pluri-sémiotiques en contexte de formation, les situations de formation «sont traversées par un certain nombre d'évolutions significatives, qui méritent d'être analysées pour une meilleure compréhension des situations de formation et des mécanismes cognitifs à l'œuvre dans ces situations, mais 
aussi pour un réexamen de questions linguistiques déjà bien balisées dans ce nouveau contexte ".

3 Nous nous appuierons sur trois séquences pédagogiques d'interactions en ligne entre un tuteur francophone et un apprenant allophone. Nous avons choisi de montrer des séquences prises dans le feu de l'interaction pédagogique qui sont problématiques et qui témoignent d'une difficulté dans l'agir professionnel de jeunes enseignants qui font leurs premières armes dans ce contexte si particulier d'un enseignement à la fois à distance et en face-à-face. Auparavant, nous rappellerons le statut et le rôle que les stratégies de reformulation ont $\mathrm{pu}$ avoir dans la professionnalisation du domaine du français langue étrangère (désormais FLE).

\section{Retour sur la notion de reformulation dans le domaine du FLE}

S'il est communément admis que les travaux en linguistique sur la reformulation débutent dans les années quatre-vingts (cf. Fuchs 1982, Gülich et Kotschi 1983, 1987), on peut poser d'emblée que dans le domaine du FLE la réflexion didactique sur la reformulation et la paraphrase est bien antérieure. C'est pourquoi nous adopterons une perspective chronologique. En effet, cette stratégie discursive qui fait partie de l'arsenal professionnel de l'enseignant pour faire accéder au sens - sans passer par la traduction en LM - prend corps dans la période qui voit l'essor de la méthodologie audiovisuelle. La reformulation, glosée par Henri Besse (1985 : 71) comme une traduction intralinguale, est, en effet, l'une des cinq démarches préconisées « pour faire comprendre ${ }^{4}$ » dans la préface du manuel Voix et images de France qui a fait l'objet de six années d'expérimentation avant sa publication définitive en 1961.

5 Pour Janine Courtillon (1972:5) qui développe « Quelques aspects de l'accès au sens dans la classe audiovisuelle », la paraphrase est une technique de mise en évidence du signifié. Elle distinguera ainsi une paraphrase par analogie ("Catherine va chez elle = elle rentre »), une paraphrase par opposition, antithèse («Les tasses sont sales, maintenant elles sont propres ». «Laver » est explicité comme l'équivalent de «rendre propre » à partir de « sale »), et une paraphrase définition (« femme de ménage » sera paraphrasé par : « aide au travail à la maison, n'habite pas là, travaille à l'heure »).

6 Dans un numéro spécial du bulletin Voix et images du Crédif, destiné à la présentation du manuel De vive voix (paru en 1972), Marc Argaud et Benoit Marin clarifient et précisent le statut et la fonction particulière de la paraphrase dans ce nouveau manuel :

«Quant aux paraphrases, elles seront de plus en plus conseillées au fur et à mesure que le bagage de l'élève devient plus important. Car c'est l'élève, et non le professeur, qui devra paraphraser les répliques du dialogue et montrer ainsi comment il les interprète. La paraphrase, telle que nous la préconisons, ne consiste pas seulement - ou même pas du tout - à «dire autrement » ce que dit le personnage. Elle consiste à expliciter la totalité des sens et des intentions qu'exprime un énoncé produit par un locuteur dans une situation donnée » (p.15).

7 Le déplacement est de taille puisque ce n'est plus le professeur qui paraphrase mais l'élève! Et ce déplacement est révélateur des évolutions méthodologiques qui progressivement vont accorder à l'apprenant une plus grande initiative et une autonomie alors naissantes. 
8 En 1979, Robert Galisson distinguera d'une part les paraphrases paradigmatiques (ou de substitution) qu'il subdivise encore en paraphrases synonymiques (" je m'engouffre, j'entre très vite ») et paraphrases antonymiques ("d'occasion... il n'est pas neuf ») et d'autre part, les paraphrases syntagmatiques (ou d'expansion) qui complètent ou prolongent l'énoncé de départ: "Qu'est-ce que ça veut dire tentative? Est-ce qu'ils font exploser la préfecture? Non... on a découvert l'explosif. Le fait de donner une précision supplémentaire sur la situation (on a découvert) permet aux étudiants de comprendre le sens de tentative » (cité et commenté par Francine Cicurel (1985 : 28), italiques de l'auteur).

Henri Besse, quant à lui, s'attachera à caractériser la paraphrase communicative :

"On appellera «paraphrase communicative» toutes les phrases qui dans une situation d'interlocution donnée peuvent être substituées les unes aux autres, sans que pour des locuteurs-auditeurs compétents dans la langue-culture concernée, l'intention de communiquer qu'elles expriment ou l'effet qu'elles peuvent produire en soit sensiblement modifié, c'est-à-dire qu'elles expriment ou produisent une intention ou un effet clairement distinct ou autre." (1985: 104 - 105).

10 Francine Cicurel et Éliane Blondel (1996) poursuivront ensuite la réflexion sur les paraphrases dans les classes de langue. À partir d'extraits de cours de FLE pour des étudiants de niveau avancé, le deuxième auteur s'intéresse aux différentes formes de la reformulation paraphrastique et souligne que les paraphrases, qui peuvent s'enchainer ou s'enchâsser, sont le plus souvent auto-initiées et auto-reformulées par l'enseignant, donc non soumises à une négociation verbale avec les apprenants. Son analyse porte sur les variations paraphrastiques récurrentes et sur les glissements sémantiques qui s'opèrent entre l'énoncé source et le reformulant. Eliane Blondel distingue ainsi les reformulations soumises à une modification dans les opérations énonciatives (ex : nouveau repérage temporel, déplacement des marques personnelles) et celles entrainant des modifications lexicales.

11 Si l'on a pu dire que la reformulation est un acte de langage à visées multiples, on voit que dans le cadre de la formation en didactique des langues elle prend une dimension particulière. En effet, la finalité de la mise en équivalence n'est pas comme dans une interaction endolingue, où l'on peut supposer une compétence linguistique relativement équilibrée chez les interlocuteurs, une quête du " mieux-dit dans du déjà dit » (Le Bot et al . 2008). En classe de langue, ce qui apparait comme primordial c'est l'aboutissement de la compréhension: c'est donc la valeur pragmatique de la reformulation qui se trouve constamment en ligne de mire de l'enseignant... Ainsi l'exemple célèbre de Francine Cicurel (1994: 108) «pschitt... » pour illustrer une action rapide comme celle de prendre un taxi au vol n'est pas forcément du « mieux-dit » mais c'est du plus efficace du point de vue de la communication pour faire accéder au sens. La spécificité de la reformulation en classe de langue est peut-être son caractère polymorphe car le triptyque classique « énoncé source / marqueur / énoncé reformulé » s'y actualise de manière très variable : la reformulation peut aussi avoir la fonction de feedback, prendre la forme d'une répétition en écho...

\section{Multimodalité de la communication en ligne}

12 La situation d'un cours par visioconférence institue un contexte plus spécifique encore qu'une classe de langue. Charlotte Dejean-Thircuir et François Mangenot (2006) ont mis en évidence la spécificité et l'ambigüité énonciative de la communication dans ce type de 
dispositif à mi-chemin entre une conversation informelle entre pairs et un dialogue inscrit dans un format contraint par une visée acquisitionnelle. Dès lors le statut de la parole des étudiants-tuteurs oscille sur un axe dont l'un des extrêmes se caractérise par un dialogue + figé + fictionnel (selon l'exemple, resté célèbre, de l'enseignant qui demande l'heure, et qui veut seulement vérifier si l'apprenant sait dire l'heure...) alors qu'à l'autre extrême on aurait un dialogue + informel + spontané + personnel.

Nous abordons dans cette étude la paraphrase reformulative en la couplant à la notion de multimodalité. Cette notion, très en vogue dans les pays anglo-saxons (Oviatt 1999, Kress \& Van Leeuwen 2001, Kress 2004) inscrit la communication dans une approche sémiotique et sociale, allant au-delà du matériau linguistique et fédérant tous les moyens de construire du sens. En contexte francophone, la notion de multimodalité peut être considérée comme un concept nomade qui est investi par diverses disciplines: de la géographie (Stéphane Autran (2009) considère que le centre d'échanges de Perrache à Lyon, est le lieu d'invention de la multimodalité) à l'informatique (Bellik \& Teil, 1992) en passant par les recherches à orientation ethnométhodologique (Mondada 2005), entre autres. Dans le domaine de la didactique des langues, on a pu faire le constat de l'extension et de l'ambiguïté de cette notion «susceptible d'évoquer la multiplicité des voies (mimo-gestuelle, proxémique, verbale...) que peut emprunter la communication humaine pour se manifester, et qui relève donc d'une analyse de la discursivité, mais aussi la diversité de moyens ou supports technologiques de cette même communication (audio, asynchrone écrit (forums), synchrone écrit (chat / clavardage), vidéoconférences, ...)» (Marquilló Larruy 2012: 67 - 68). Cette notion trouve ainsi une résonnance particulière dans les travaux de Thierry Lancien ${ }^{5}$ (2000), Marie-José Barbot (1997) ou encore Christine Develotte et al. (2011) qui ont développé une réflexion très proche de celle menée dans le domaine de la multimodalité, à propos de l'utilisation pédagogique du multimédia. Ce type d'explicitation renvoie à des démarches qui cherchent à prendre en considération la communication comme un phénomène total, ce que Jacques Cosnier (2011) appelle le "totexte", représenté par une modélisation qui englobe les éléments 
voco-verbaux (le texte) et posturo-mimo-gestuels (le cotexte), comme explicité dans le schéma ci-dessous.

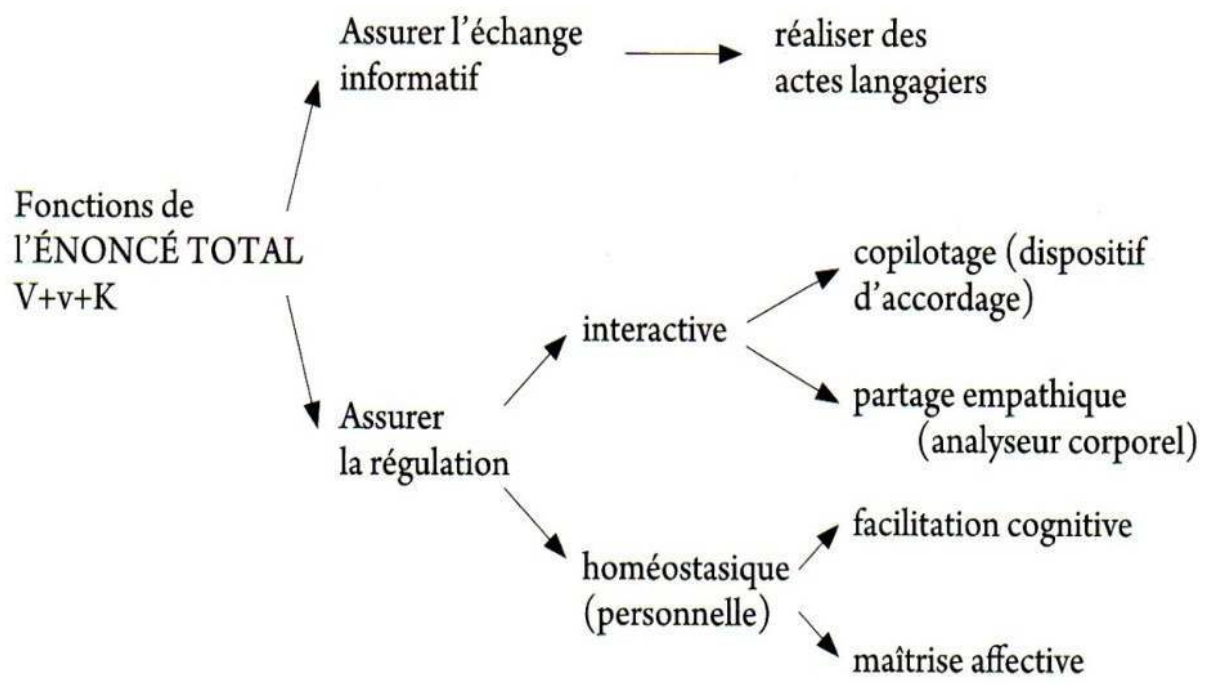

Schéma - Les fonctions du totexte

1. Modèle de Cosnier (p. 50) : les fonctions de l'énoncé total ou totexte

14 Notre étude renvoie davantage aux différents aspects de la multicanalité (texte, image, son) qui entrent en jeu lors de la conversation en ligne. S'intéressant à la gestualité qui est développée dans ce type de conversation, Jacques Cosnier (ibid.) note que ce dispositif, qui ne donne un accès direct qu'à la tête et au buste du partenaire, induit une gestuelle spécifique chez les interlocuteurs. Les expressions faciales tiennent en effet une place majeure dans la régulation de l'interaction et elles sont plus nombreuses que dans les interactions dites ordinaires.

Les séquences pédagogiques que nous allons décrire et analyser par la suite sont représentatives de ce type d'interactions dans lesquelles des facteurs techniques peuvent parasiter l'échange et faire dévier l'intention de communication. Elles sont également représentatives de la difficulté, pour de jeunes enseignants novices, d'instaurer des stratégies reformulatives efficaces qui doivent résoudre une double contrainte: faire accéder à la compréhension sans entraver pour autant la dynamique discursive.

\section{Spécificités du dispositif et méthodologie de recueil des données}

L'originalité de notre dispositif peut être déclinée à plusieurs niveaux.

17 D'une part, dans les choix techniques qui ont des conséquences tant du point de vue pédagogique que du point de vue du stockage et de la consultation des données pour la recherche. Le dispositif mis en place par Christophe Naud, responsable de l'ingénierie pédagogique à la Maison des langues de l'université de Poitiers, répond en effet à la double contrainte d'une connexion aussi simplifiée que possible (ordinateurs PC, casques avec micro, Webcam, Internet) qui associe Adobe Connect (choix de l'université de Poitiers pour ses visioconférences) et l'utilisation de logiciels libres de droits (Audacity pour 
l'audio et Debut Video Capture pour la capture vidéo de l'écran). Ainsi des archives audio, vidéo et textuelles (messagerie instantanée) ont pu être réalisées.

D'autre part, du point de vue de la mise en place du dispositif de formation des futurs enseignants de français puisqu'on a tiré parti du déséquilibre entre le nombre de tuteurs (30 étudiants de master 2) et le nombre de formés (11 apprenants de FLE). Il s'agit donc d'une interaction complexe -bien que réglée- puisque chacun des alloglottes est mis en relation avec trois tuteurs lors de chaque connexion. Ensuite, sans pour autant les imposer, on a également décidé de ne pas écarter la possibilité d'échanges asynchrones via un blog créé dans le cadre d'un cours connexe qui forme les étudiants à l'utilisation des supports multimodaux issus d'internet.

Par ailleurs, du point de vue de la formation associée à la recherche, les étudiants du master auront tenu un journal de bord qui aura fait l'objet d'une réécriture synthétique après la fin de l'expérimentation. Ils auront par la suite été sensibilisés, dans le cadre d'un cours portant sur les spécificités des modalités discursives/interactionnelles de la classe de langue, aux problèmes de transcription et d'analyse de ces corpus multimodaux en vue de la recherche et de l'étayage de leur propre formation pédagogique. Les pratiques tutorales auront donc fait l'objet d'une rétrospection et d'une auto-évaluation.

Originalité, enfin, du point de vue des apprenants alloglottes qui évoluent d'ores et déjà en milieu francophone, bien que suivant la totalité de leurs cours en anglais et échangeant au quotidien principalement dans cette langue ou dans leur langue maternelle. Il s'agit en effet de la première expérimentation du F1L menée entre deux établissements en France, alors qu'auparavant l'établissement des apprenants était situé en milieu non francophone. Ceci laisse supposer des échanges interculturels riches avec les tuteurs, les alloglottes appartenant à différentes langues/cultures (Vénézuela, Chine, Mexique, Espagne, Roumanie) et ayant un contact direct avec la culture française. Les thématiques des tâches à concevoir par les tuteurs (et préparées collectivement) ont été définies en fonction des besoins professionnels (recherche de stage, CV, lettre de motivation, système éducatif français) et quotidiens (vie des jeunes en France, stéréotypes) des apprenants.

21 Du point de vue de la méthodologie de la recherche, nous avons fait le choix d'une triangulation des données en nous basant sur les enregistrements (audio-vidéo ou audio seulement) des interactions, le recueil des échanges écrits synchrones via chat et asynchrones sur le blog, les synthèses réflexives des tuteurs rédigées quelques semaines après la fin de l'expérimentation ainsi qu'un questionnaire soumis aux tuteurs et aux apprenants afin de recueillir leurs perceptions sur ces échanges.

Le tableau suivant résume les caractéristiques de chaque groupe de participants lors des 6 séances (30 mn chacune) qui ont eu lieu.

\begin{tabular}{|l|l|l|l|l|l|l|l|}
\hline & Institution & $\begin{array}{l}\text { Niveau } \\
\text { académique }\end{array}$ & $\begin{array}{l}\text { Niveau } \\
\text { langagier }\end{array}$ & Age & $\begin{array}{l}\text { Pays } \\
\text { d'origine }\end{array}$ & Nombre & Répartition \\
\hline
\end{tabular}




\begin{tabular}{|c|c|c|c|c|c|c|c|}
\hline $\begin{array}{l}\text { Apprenants } \\
\text { de FLE }\end{array}$ & $\begin{array}{lr}\text { Telecom Sud } \\
\text { Paris } & \text { et } \\
\text { Telecom } & \\
\text { Ecole de } \\
\text { management } \\
\text { (Evry) }\end{array}$ & $\begin{array}{l}\text { Master of } \\
\text { science }\end{array}$ & B1 & $\begin{array}{l}20-25 \\
\text { ans }\end{array}$ & $\begin{array}{l}\text { Vénézuela, } \\
\text { Chine, } \\
\text { Mexique, } \\
\text { Espagne, } \\
\text { Roumanie }\end{array}$ & 11 & 1 apprenant \\
\hline $\begin{array}{l}\text { Apprentis- } \\
\text { tuteurs de } \\
\text { FLE }\end{array}$ & $\begin{array}{l}\text { Université } \\
\text { de Poitiers }\end{array}$ & $\begin{array}{l}\text { Master } 2 \text { de } \\
\text { didactique } \\
\text { des langues } \\
\text { et du FLES }\end{array}$ & $\begin{array}{l}\text { natifs / C1 } \\
\text { (étudiants } \\
\text { internatio- } \\
\text { naux) }\end{array}$ & idem & $\begin{array}{l}\text { France, } \\
\text { Algérie, } \\
\text { Mali, } \\
\text { Russie, } \\
\text { Colombie, } \\
\text { Corée, } \\
\text { Japon, } \\
\text { Etats-Unis }\end{array}$ & 30 & $\begin{array}{l}1 \text { tuteur + } \\
2 \\
\text { observateurs } \\
\text { à tour de } \\
\text { rôle }\end{array}$ \\
\hline
\end{tabular}

2. Profil des participants au dispositif du F1L Poitiers-Evry (2011-12)

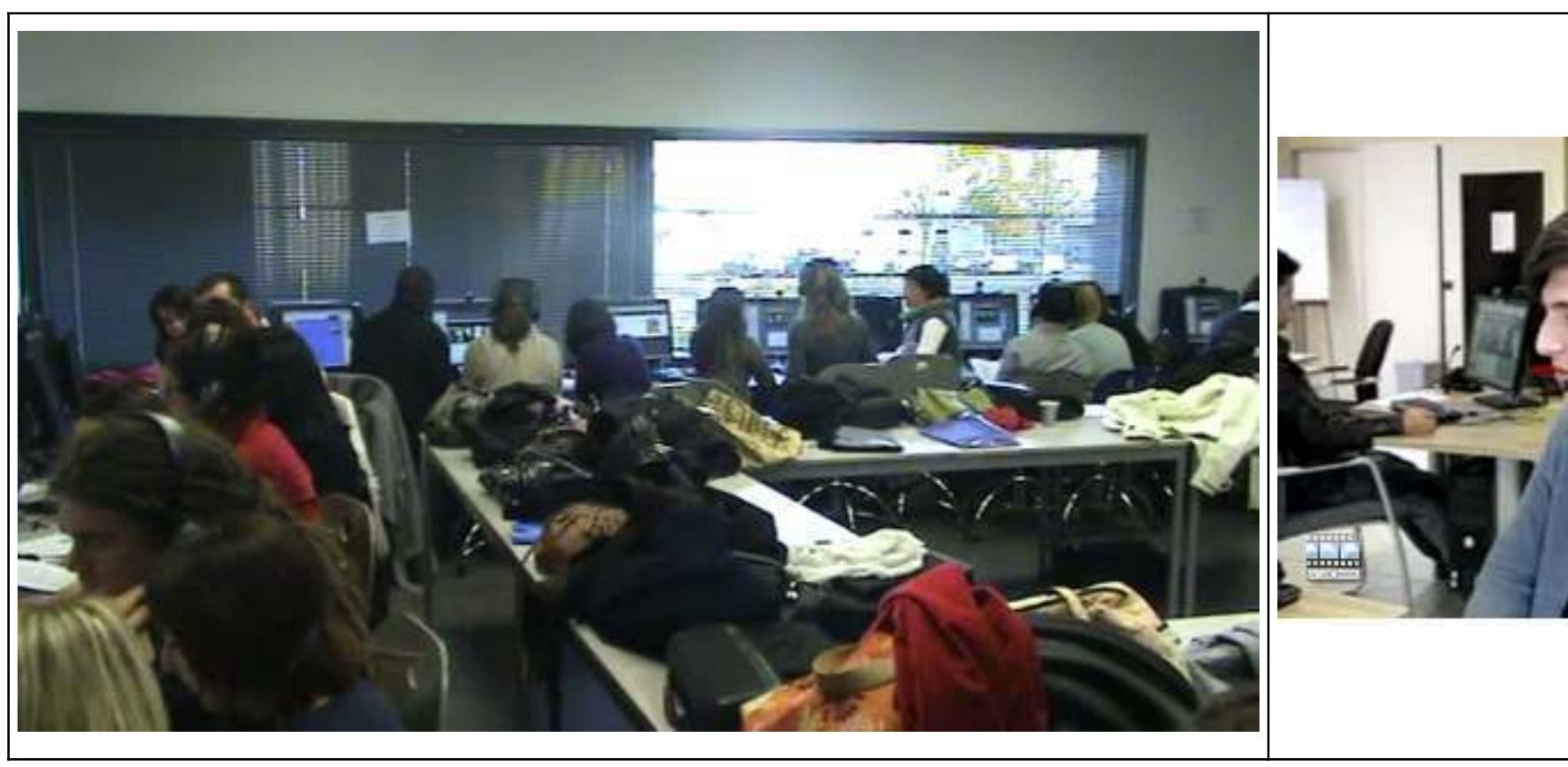

3. Dispositif de visioconférence à Poitiers 4. Dispositif de visioconférence à Évry

23 On se focalisera ici sur l'analyse des échanges oraux et écrits synchrones et l'on verra quelles sont les stratégies reformulatives adoptées par les tuteurs (et les apprenants dans une moindre mesure) pour assurer l'efficacité communicative. En s'intéressant à trois séquences pédagogiques particulièrement représentatives des usages de la multimodalité au service de la reformulation, on s'attachera à la manière dont sont résolus les problèmes d'intercompréhension entre participants. La première séquence illustre la richesse des variantes reformulatoires de la tutrice pour faire accéder au sens à l'aide d'un support iconique, tandis que les deuxième et troisième séquences montrent le rôle du non verbal (gestuelle et clavardage) accompagnant les procédés explicatifs ${ }^{6}$ (pour accéder aux enregistrements vidéo correspondant aux analyses, cliquer ici) 


\section{Analyse de séquences conversationnelles}

\subsection{Séquence $1:$ "T'es où ? »: reformulation et variation paraphrastique}

Dans cette séquence assez longue (presque $4 \mathrm{mn}$ ) consacrée aux études, la tutrice demande à une apprenante sinophone de situer son niveau académique sur un schéma qui a servi de déclencheur à la séance (cf. image 5). L'apprenante vérifie au tout début de la séquence s'il s'agit de situer son niveau actuel («maintenant ?») mais la tutrice n'entend pas sa question et à partir de là, l'échange s'enlise et piétine autour de la question du choix d'un niveau académique. Cette séquence est emblématique de la variété des reformulations de la tutrice pour assurer l'intercompréhension. À partir de la question initiale « tu te situes où sur ce schéma? », on relève les variantes suivantes:

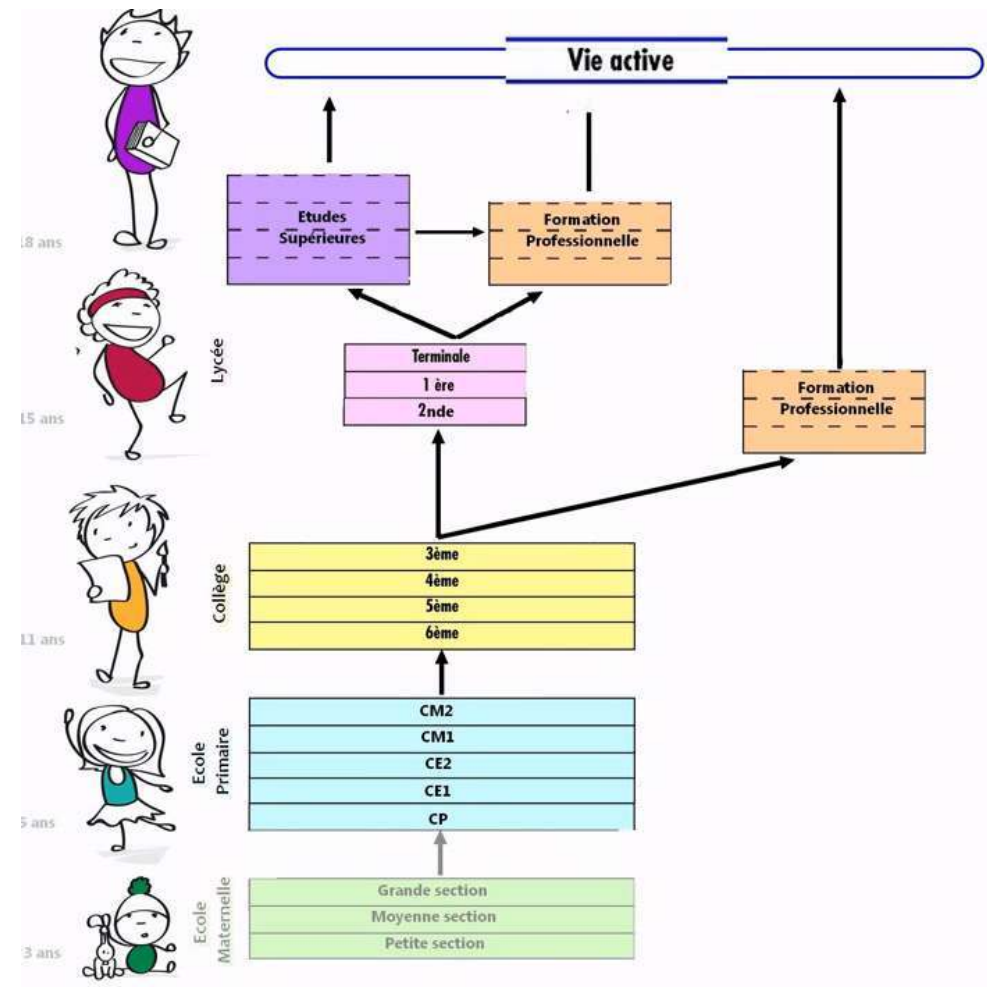

5. Support iconique utilisé pour le thème des études en France

t'es où toi ?

dans quelle étape tu es?

où tu te situes? ici?

tu es dans quelle étape?

où j'peux t'trouver?

t'es où si je monte?

t'as choisi?

pourquoi t'es en France? tu fais quelles études?

6. Variantes reformulatives de la question "tu te situes où sur ce schéma?"

La reformulation parasphrastique qui s'opère de a) à f) se caractérise par une équivalence sémantique entre la question de départ et tous ces énoncés. Les deux dernières variantes s'éloignent néanmoins de l'énoncé source X ("tu te situes où sur ce schéma ?") et relèvent 
plutôt de la reformulation non paraphrastique (cf. Roulet, 1987) : l'énoncé Y (reformulant) présente une identité uniquement référentielle et non sémantique avec $\mathrm{X}$. On vise toujours le même référent, à savoir le lieu où se situe l'apprenante dans son parcours scolaire, mais $\mathrm{X}$ et $\mathrm{Y}$ n'ont plus le même sens dénotatif. Après plusieurs échecs d'intercompréhension, la tutrice s'éloigne ainsi du paradigme d'origine pour poser la question sous un autre angle : "t'as choisi ?" en g) reprend la thématique du choix d'études abordé par l'apprenante (A) dans l'énoncé qui précède, sans succès. La dernière reformulation en h) guide l'apprenante, en deux temps, vers l'objet de son séjour en France en renvoyant implicitement à "tu fais des études supérieures". Comme on le voit dans l'extrait ci-dessous, la tutrice $(\mathrm{T})$ revient finalement à sa question d'origine et clot la séquence en répondant elle-même à cette question par " $t$ 'es dans la partie études supérieures".

\begin{tabular}{l} 
A36: (...) si si on choisit le lycée on doit apprendre trois ans le première année à lycée le \\
deuxième année le fin le fin année à lycée après on choisit le + on peut choisir peut-être le \\
études sup-supérieures / \\
\hline T37: et toi TOI maint'nant tu as choisi c'est bon + hein $\uparrow$ \\
\hline A38: ouais \\
\hline T39: toi t'as passé t'as passé le baccalauréat t'as choisi $\uparrow+$ \\
\hline A40: ouais j'ai choisi/ \\
\hline T41: alors t'es où $\uparrow$ pourquoi t'es en France tu fais quelles études $\uparrow$ \\
\hline A42: ah j'ai choisi le le le le le le étude dure (rire) j'ai choisi le / \\
\hline T43: donc donc t'es t'es dans la partie études supérieures c'est ça $\uparrow$ \\
\hline A44: ouais c'est ça \%M je l'ai choisi + je sais c'est dur mais je l'ai choisi
\end{tabular}

\section{Extrait clôturant la séquence "t'es où ?"}

Au cœur de la démarche explicative de la tutrice, on note une stratégie de pointage et de monstration (pour situer le niveau sur le schéma) qui rapproche ce dispositif de ce que l'on peut aisément retrouver dans une interaction de type didactique en face-à-face. Par ailleurs, un malentendu lié à la compréhension du mot schéma est résolu quant à lui grâce à l'intervention de la deuxième tutrice du groupe, d'où l'intérêt du rôle d'observateurs « actifs » joué par les autres membres du trio de tuteurs. En effet, ces tuteurs en retrait de l'échange sont souvent plus à mêmes de repérer les moments critiques et les éléments du code posant des problèmes d'interprétation de la part des participants, puis d'intervenir à bon escient pour aider le tuteur menant l'interaction. Une vraie synergie entre tuteurs se met alors en place et les groupes collaborant le mieux sont également les plus efficaces du point de vue de l'étayage (Bruner, 1983).

«Le rôle d'observateur est tout aussi important que celui de tuteur car il permet d'avoir un regard différent sur la séance pédagogique en cours et le recul nécessaire sur l'attitude et le guidage pédagogique à tenir afin d'améliorer ses propres stratégies d'enseignement. » (Adeline) 
« En tant qu'observatrice c'est très intéressant de voir les réactions de l'apprenant et du tuteur. On peut plus facilement se rendre compte et analyser les problèmes rencontrés par les deux participants. » (Marjorie) document vidéo qu'il vient de visionner, la tutrice lui demande s'il a reconnu quelqu'un. Cet énoncé de départ est aussitôt reformulé sous la forme d'une répétition marquée par un ralentissement et une pause entre «reconnu» et le modalisateur «peut-être». L'apprenant a alors une sorte d'acquiescement de complaisance sans pour autant répondre à la question. La tutrice reformule ensuite sa question initiale en faisant appel au verbe synonyme « démasquer » (« est-ce que tu l'as démasqué?»).

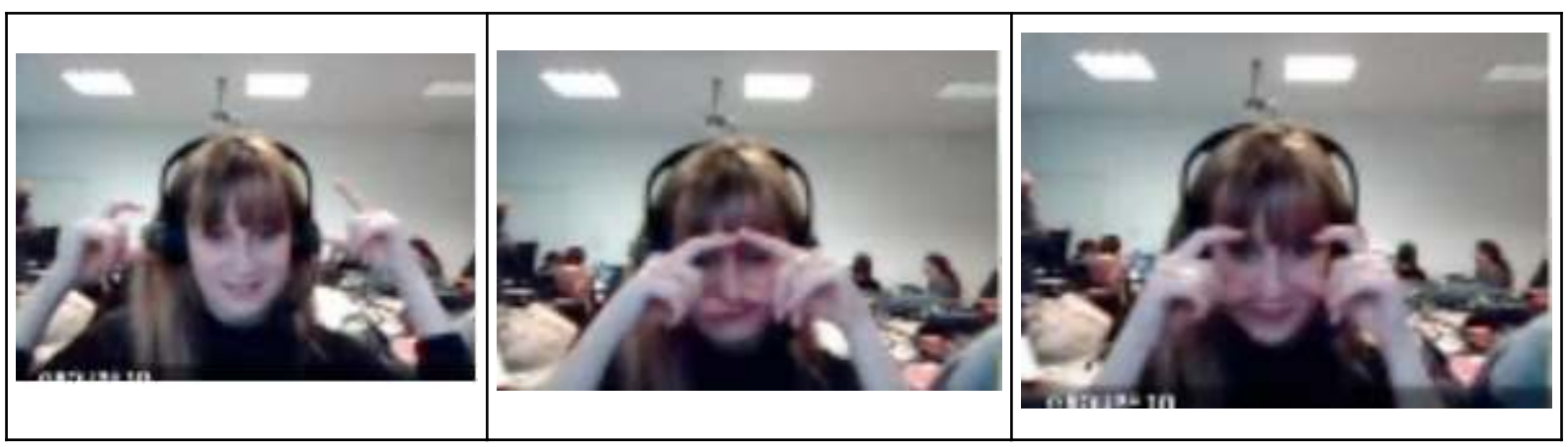

8. Gestuelle de la tutrice visant à illustrer l'expression « démasquer » (enlever le masque)

S'ensuit alors ce qu'on appelle en linguistique interactionnelle une "séquence latérale » (Jefferson, 1972) autour de cette expression : l'apprenant manifeste son incompréhension par une interrogation ( «quoi? ») et la tutrice répète à plusieurs reprises la question à l'identique, sans succès. Elle change alors de stratégie et passe par une définition littérale de l'expression (démasquer $=$ enlever le masque) tout en accompagnant sa verbalisation d'un geste illustratif co-verbal et kinémimique (mouvement de retrait du masque) (Cosnier 1987, Tellier \& Stam 2012). Mais ce geste n'aide pas à la compréhension, ce qui entraine une nouvelle stratégie explicative par le biais du clavardage.

31 Ce dernier se décompose en 2 mouvements : lors du premier, la tutrice tape «démasquer quelqu'un», comme si elle isolait l'énoncé faisant l'objet d'une explication (comme une 
entrée de dictionnaire) et dans un deuxième temps, elle introduit par le marqueur « ça veut dire » - qu'elle oralise en même temps qu'elle l'écrit - une paraphrase définitionnelle : «reconnaître quelqu'un» ce qui fait revenir à l'énoncé de départ. Cette stratégie aboutit puisqu'on note cette fois-ci un acquiescement appuyé de l'apprenant. La tutrice s'appuie sur cette marque de compréhension pour revenir sur ce qui n'a pas été compris. Le retour à l'énoncé incompris est réalisé par un marqueur discursif de justification (" parce que ») avec une reformulation en abyme qui décompose le verbe « dé-masquer » en reformulant le privatif par « enlever ». La gestualité double la reformulation et on pourrait parler ici d'une reformulation gestuelle car le geste est lui-même décomposé ; du point de vue verbal on a une cascade de répétitions entrecoupée par une alternance codique (" máscara»), avant une dernière reformulation conclusive sous forme de traduction intralinguale (" tu enlèves le masque et tu l'as démasqué »). L'apprenant acquiesce à nouveau et manifeste enfin une compréhension effective de l'élément " démasqué ».

Du point de vue de l'efficacité communicative, il faut noter que cette sorte de redondance pédagogique et de surexplicitation brouillent l'échange. On assiste ici à une dérive explicative : la reformulation opacifie plus qu'elle n'éclaircit (dans ce contexte démasquer est moins transparent que reconnaitre). De plus, l'explicitation ultérieure ne permet pas à la conversation de continuer ou d'aboutir : lorsque la tutrice a eu la confirmation de la compréhension du terme démasqué, elle fait bifurquer l'échange vers un autre sujet. On se demande alors quelle était la finalité, du point de vue de l'apprentissage linguistique, de cette surenchère... Interrogée à ce propos, quelques mois après cette séquence, et après l'avoir revisionnée, la tutrice indique s'être trouvée empêtrée dans une explication dont elle avait bien vite senti le caractère inapproprié mais une fois lancée, elle s'était sentie obligée d'aller jusqu'au bout... (sur les rétrospections, voir les travaux de Nicolas Guichon, 2011 et José Ignacio Aguilar Rio, 2011).

\subsection{Séquence 3 : « Ça fouette ! » : reformulation et rôle du clavardage}

34 Cette troisième séquence est extraite de la dernière séance de l'échange en ligne entre un autre apprenant hispanophone et trois autres tuteurs francophones, dont deux observateurs. Elle a pour thème les langues, l'un des objectifs étant de comprendre et partager des expressions françaises du langage familier. La séquence met là encore en évidence la lenteur d'une convergence pédagogique entre les acteurs de l'interaction malgré diverses stratégies mises en place pour atteindre l'intercompréhension.

Le tuteur qui mène l'interaction, R., a choisi de présenter une de ses expressions favorites : «ça fouette ». Il annonce l'activité en présentant l'expression cible qu'il accompagne d'une gestuelle adaptée (balayage devant le visage). Constatant que son geste ne suffit pas à déclencher une marque de compréhension chez l'apprenant, le tuteur fournit de l'étayage en donnant des exemples sous forme de mise en situation (ex: "si je porte les mêmes chaussettes depuis 3 semaines, je vais dire "ça fouette"»), et en faisant appel au clavardage (notation-répétition graphique de l'expression). Puis il prend pour argent comptant l'acquiescement de l'apprenant à sa question : «tu as compris? ». La séquence explicative semble alors terminée. 


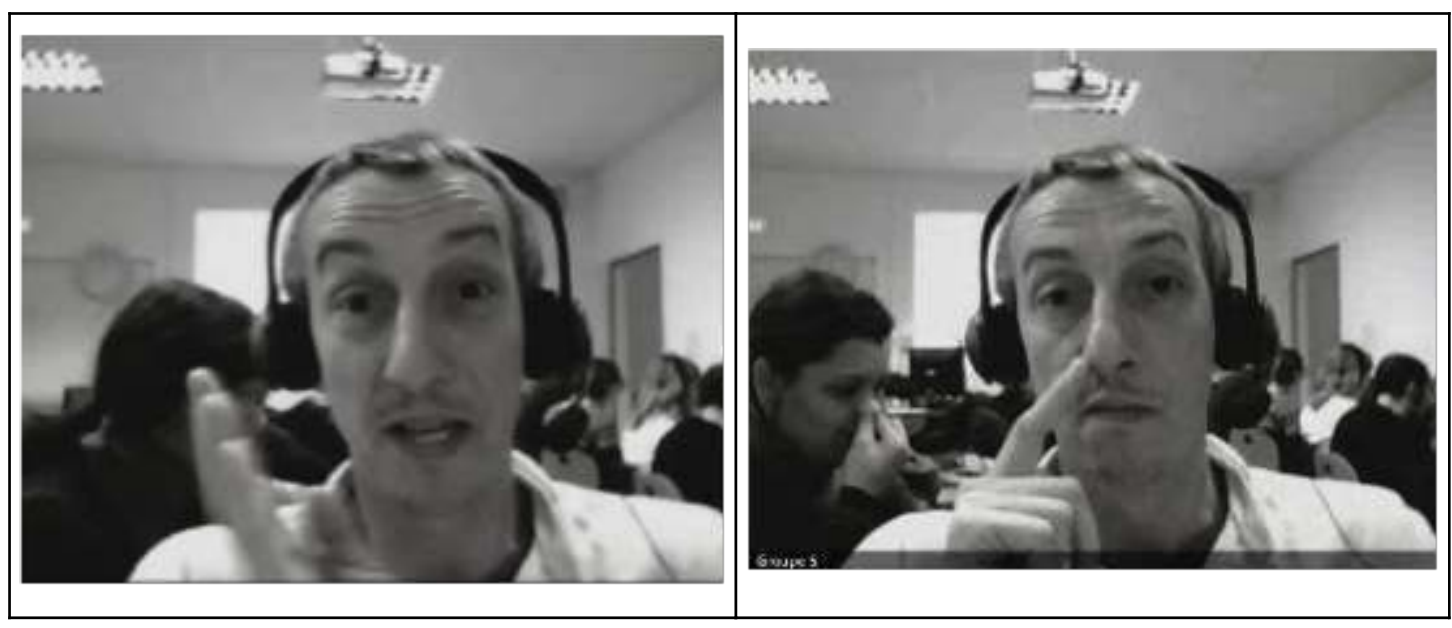

9 et 10. Gestes des tuteurs visant à illustrer les expressions « ça fouette » (9) et « ça sent mauvais » (10)

Mais survient alors un élément qui va déséquilibrer la situation lorsque le deuxième tuteur du groupe, observateur jusque-là, va donner l'équivalent "ça sent mauvais » par clavardage. L'apprenant ne comprend pas cette expression, qu'il a d'ailleurs du mal à prononcer (/sasenmuvo/), tandis que le tuteur pris dans l'interaction et son scénario pédagogique reste sourd à cette incompréhension. Enfin l'échange avec le tuteurobservateur va permettre à R. d'identifier le malentendu. Les deux tuteurs vont alors tenter des reformulations : une fois la cause de l'incompréhension repérée, une première reformulation passe par le biais du clavardage (" ça pue »). Le tuteur, après avoir découpé l'énoncé problématique, passe par l'antonyme en faisant appel à du métalangage (" l'inverse de bon »), puis reformule avec une expression synonyme qui lui parait plus simple: «pas bon ». Finalement le rééquilibrage ne sera atteint que lorsque le tuteur intègrera cette expression dans un énoncé reformulateur établissant une équivalence sémantique ("ça sent mauvais = ça sent pas bon ») et que s'y ajoutera une gestuelle plus "transparente".

On note en effet que les gestes et les expressions du visage ne sont pas aussi compréhensibles que dans une classe de langue car le temps de décalage des interactions en ligne crée un brouillage des gestes trop rapides. De plus, le champ de vision est restreint par le cadre de la caméra. Le geste illustratif du tuteur (balayage devant le nez, cf. image 9) est peu visible à l'écran et il est difficilement interprétable. On peut supposer que la variable culturelle intervient également dans la difficulté à décoder ce type de gestes spécifiques.

Par contre, les gestes fixes sont plus efficaces car plus visibles, comme celui du doigt sur la narine. On note dans cette même séquence un autre geste du troisième tuteur en arrière-plan (se boucher le nez) (cf. image 11) qui a sans doute davantage facilité la compréhension en créant de la redondance par rapport au langagier. Quant aux mimiques faciales, qui reflètent la mobilité et l'expressivité des visages propres à la personnalité de chacun, elles semblent jouer un rôle encore plus important que les gestes à proprement parler. 


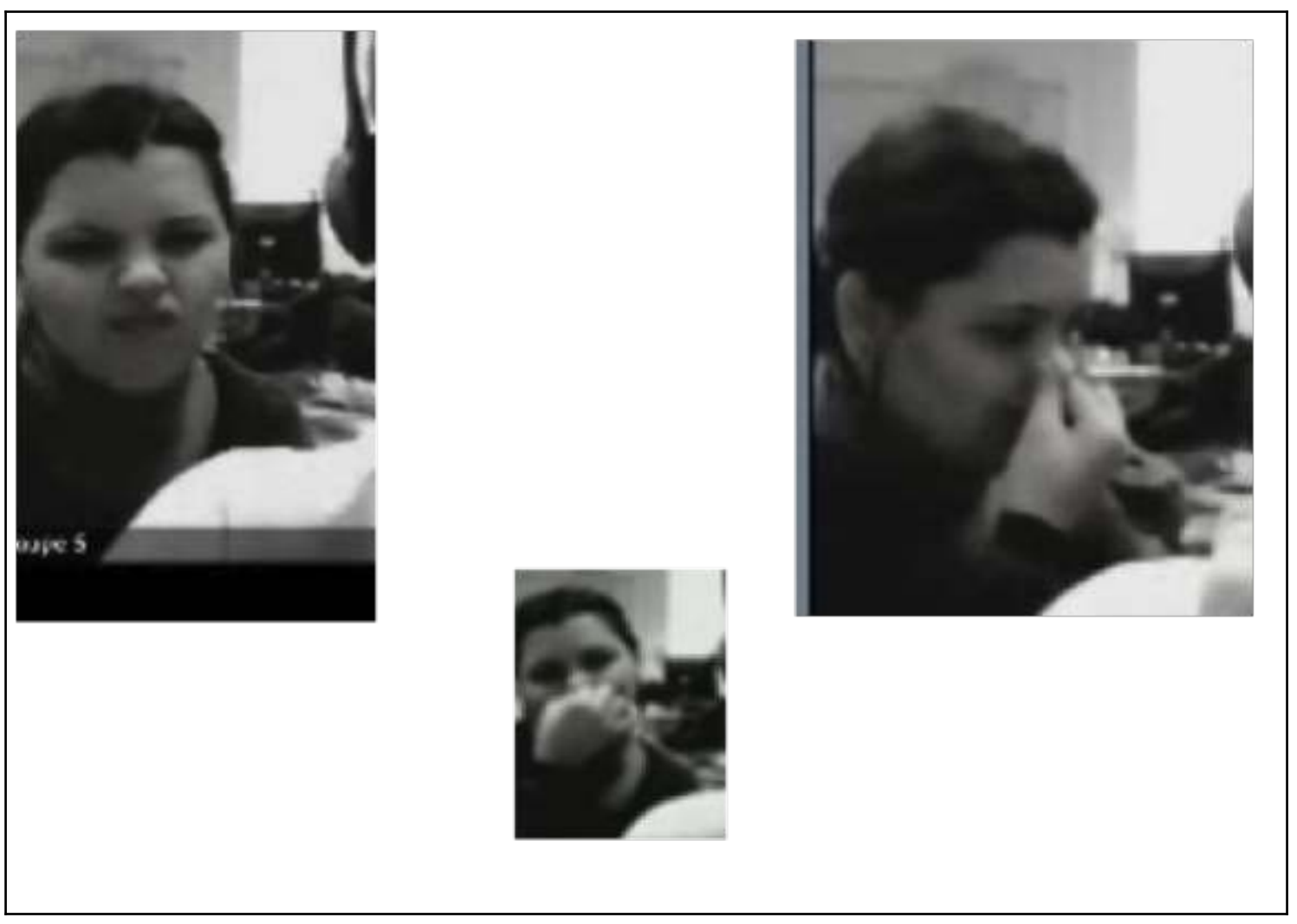

11. Mimiques et gestuelle de la tutrice observatrice pour l'expression « ça sent mauvais »

La posture mimico-gestuelle de l'apprenant peut être un bon indicateur de l'état de sa compréhension : les tuteurs apprennent alors à surveiller cet aspect non verbal de la communication pour interpréter les réactions de l'alloglotte et ajuster leurs régulations pédagogiques. Certains d'entre eux y sont d'ailleurs particulièrement réceptifs :

«De plus, notre apprenant s'appuyait beaucoup sur les reformulations et le non verbal. A mon avis, l'intérêt dans notre expérience, à ce niveau est d'avoir appris comment repérer les difficultés et les incompréhensions de notre apprenant à travers ses mimiques et ses gestes transmis par la webcam. (Exemple: porter la main vers le casque me semble typique de la visioconférence). Ceci nous a permis de remédier aux difficultés énoncées implicitement ». (Kiria)

Du côté des reformulations de l'apprenant (A.), dont un extrait est présenté ci-dessous, on constate une certaine hétérogénéité de formes paraphrastiques concurrentes pour l'expression d'un même concept, qui peut se traduire par des marques transcodiques quand ce dernier est en situation de recherche lexicale. Pour négocier le sens de l'expression "ça sent mauvais ", A. fait appel à tout un arsenal de stratégies de résolution des problèmes de communication (cf. Bange 1992), allant des stratégies de substitution aux stratégies de réalisation des buts de communication. Les stratégies de substitution se manifestent ici par le recours à la L1 $\left({ }^{*}{ }^{*}\right.$ como*») ou une autre LE ( ${ }^{*}$ angry*») pour compenser des savoirs insuffisants ou incertains dans l'interlangue. Le passage par une autre langue sous la forme d'alternances codiques dans la production langagière en L3 (cf. Rast et Trévisiol 2006, Bono 2007, De Angelis 2007) permet à l'apprenant de se servir de ses connaissances linguistiques antérieures tout en allégeant la charge cognitive induite par la tâche : l'anglais, langue d'enseignement et de communication la plus utilisée dans 
le contexte d'apprentissage de A., lui sert de langue-pont pour tester des hypothèses sur le fonctionnement de la langue cible. Quant aux stratégies de réalisation, et plus spécifiquement d'élargissement des savoir-faire dans l'interlangue, elles peuvent prendre comme ici la forme de paraphrases de divers types en vue de négocier le sens: approximations (" pas de /bonumor/ que'qu'chose comme ça »), périphrases, synonymes (" mécontent») (cf. Gülich, 1986).

41 A. /sasenmuvo/ ça c'est *como* être ++ mmmh ++ *angry* $\uparrow$ euh chais pas comment on $\operatorname{dit~ça~/movo/~c'est~*como*~}{ }^{+++}$peut-être que c'est pas /debonumo/ $\uparrow$

R. du $\uparrow+$ tu peux répéter $\uparrow$

A. /debonumor/ que'qu'chose comme ça $\uparrow$

R. ah de bonne humeur $\uparrow$

A. oui c'est c'est mécontent $\uparrow$

12. Reformulations de l'apprenant autour de l'expression «ça sent mauvais » (/sasenmuvo/)

Dans une perspective interactionniste de l'acquisition, plus axée sur les procédés de la communication exolingue que sur les processus intrapsychiques (cf. Lüdi 1986, Vasseur 1991), la reformulation en LE joue un rôle de premier plan puisqu'elle permet à l'apprenant de communiquer avec les moyens dont il dispose tout en gérant les problèmes d'intercompréhension. Par cette stratégie de prise de risques langagiers, il déploie son interlangue dans des conditions favorables au déclenchement de l'étayage du locuteur natif : celui-ci peut lui fournir, si besoin, l'item lexical recherché. Le travail sur la langue qui est alors effectué à travers ces séquences latérales de reformulation peut parfois déboucher sur des séquences potentiellement acquisitionnelles (cf. De Pietro, Matthey et Py, 1989) lorsque l'apprenant effectue une prise/saisie à partir de l'intervention de l'interlocuteur natif.

\section{Conclusion}

À l'issue de ces analyses, quelles sont les variétés reformulatives qui se donnent à voir selon les typologies spécifiques de la didactique du FLE que nous avons évoquées dans la première partie de cette contribution? La traduction intralinguale est utilisée dans la séquence 2 (démasquer = enlever le masque) mais aussi la stratégie de traduction interlinguale ( mascara ») si tant est que l'on puisse considérer que la traduction est une variété de paraphrase. Les séquences 2 et 3 utilisent la technique de mise en évidence du signifié : paraphrase par analogie (ex: "/sasenmuvo/ ça c'est *como* être *angry*? »), par opposition / antithèse (ex: « mauvais c'est l'inverse de bon ») et paraphrase définition (ex: « démasquer quelqu'un ça veut dire reconnaitre quelqu'un»). On retrouve également des paraphrases paradigmatiques ou de substitution lorsque la tutrice de la séquence 1 dit " où j'peux te trouver Liyin, tu te situes où? ", d'autre part des énoncés comme " quand j'ai de vieilles chaussettes + quand j'ai des chaussettes que j'ai gardées pendant trois semaines j'exagère et bien ça sentira mauvais + d'accord ça va fouetter " constituent des paraphrases syntagmatiques (ou d'expansion) telles que les décrivait Robert Galisson. Enfin la séquence 1 nous donne un exemple dans lequel on oppose une reformulation paraphrastique (ex: "t'es où toi ? dans quelle étape tu es? ») et une reformulation non paraphrastique ( «' t'as choisi ? »).

Du point de vue de la multimodalité, ces trois séquences du français en première ligne entre les étudiants allophones d'Evry et les étudiants-tuteurs de Poitiers sont une illustration 
exemplaire de la complexité inhérente à ce dispositif, où geste, parole et écriture se combinent pour construire l'interaction pédagogique. Elles montrent les spécificités des reformulations dans le cadre de ce nouveau mode de communication pédagogique, médiée par ordinateur, avec l'accompagnement par plusieurs tuteurs, le recours au clavardage, les obstacles qui se présentent et les solutions efficaces trouvées par les tuteurs, notamment les mimiques, les alternances codiques.

Du point de vue de la formation professionnelle des futurs enseignants de français, les trois séquences analysées montrent la difficulté d'improviser des reformulations efficaces dans le feu d'une interaction pédagogique médiée par un dispositif de visioconférence qui n'est pas toujours facilitateur du point de vue de la fluidité communicative (temps de latence entre les messages, problème de clarté du son...). Et, si l'on a pu observer des formes de sur-intentionnalité pédagogique (le "vouloir expliquer à tout prix») qui peuvent conduire à l'effet inverse du but visé, il serait cependant extrêmement réducteur de ne retenir que cet aspect du dispositif mis en place. Car on ne peut clore cette contribution sans évoquer un bénéfice non négligeable du point de vue de la formation, à côté de l'élargissement des répertoires reformulatifs, l'apprivoisement de soi, comme on peut le voir dans ce témoignage :

«Moi, en tant que Russe, et ne partageant pas du tout la langue maternelle de l'apprenant (l'espagnol) j'ai essayé d'utiliser la reformulation pour expliciter un sujet. Tout au long des séances j'ai été donc confrontée à des demandes d'explicitations de la part de l'apprenant (José). J'ai été " contrainte », en quelque sorte, à reformuler une partie de mon discours en utilisant des synonymes, en fournissant la signification ou en utilisant le clavardage. En effet, n'ayant pas d'autre recours que le français (pas de recours possible à la traduction en espagnol, ni de recours à ma langue maternelle) j'ai fourni également assez souvent la signification d'un mot pour approfondir son explicitation ». (Natalia)

De nombreux chercheurs ont avancé qu'une dimension capitale de la compétence à enseigner est la capacité d'interpréter ce qui se passe en classe et de s'y adapter (Gaudin et Flandin 2014 : 7). Le dispositif Le français en (première) ligne, nous semble être propice à éveiller le regard professionnel de nos futurs enseignants en leur faisant prendre conscience de la diversité des modalités de l'accès au sens en langue étrangère.

\section{BIBLIOGRAPHIE}

AGUILAR RIO, J.I., (2011), « L'étude de la cognition des enseignants de langue à travers l'autoconfrontation : problématiques de recherche et précautions méthodologiques », dans Bigot V. \& Cadet L., Discours d'enseignants sur leur action en classe. Enjeux théoriques et enjeux de formation, Paris, Riveneuve, p.109-126.

ARGAUD, M. \& MARIN, B., (1973), « Une pédagogie de De Vive Voix », Voix et Images du Crédif, n²0, p.14-19.

AUTRAN, S., (2009), Le centre d'échanges de Perrache : l'invention de la multimodalité (1964 1976). http://www.millenaire3.com/uploads/tx_ressm3/Perrache_centre_echanges_01.pdf 
BANGE, P., (1992), « A propos de la communication et de l'apprentissage de L2 (notamment dans ses formes institutionnelles) ", Acquisition et Interaction en Langue Etrangère, n ${ }^{\circ}$, p.53-85.

BARBOT, M.-J., (1997), Cap sur l'autoformation : multimédias, des outils à s'approprier. Le Français dans le Monde, Recherches et Applications, Paris, Édicef, numéro spécial.

BELLIK, Y. D. \& TEIL, D. (1992), « Définitions terminologiques pour la communication multimodale ", dans Actes des 4èmes Journées sur l'ingénierie des interfaces Homme-Machine, IHM'92, Telecom Paris Publ., Paris, 30 Nov. - 2 Déc., p.229-232.

BESSE, H., (1985), Méthodes et pratiques des manuels de langue, Paris, Didier, Collection Essais. BLONDEL, É., (1996), « La reformulation paraphrastique. Une activité discursive privilégiée en classe de langue ", Les carnets du Cediscor, n4, p.47-59.

BONO, M., (2007), « La comparaison L2-L3, un tremplin vers l'acquisition trilingue », Birkbeck Studies in Applied Linguistics, $\mathrm{n}^{\circ} 2, \mathrm{p} .22-41$.

BRUNER, J.-S. (1983), Le développement de l'enfant : savoir faire, savoir dire, Paris, PUF.

CICUREL, F., (1985), Parole sur parole, Le métalangage en classe de langue, Paris, CLE international.

CICUREL, F., (1994), « Schéma facilitateur et métalangage dans l'apprentissage d'une langue étrangère ", dans Lapeyre F., Simple - Simplification, Cahiers du français contemporain, $\mathrm{n}^{\circ} 1, \mathrm{p} .103-118$. CICUREL, F. \& BLONDEL, É. (dir.) (1996), La construction interactive des discours de la classe de langue, Les Carnets du Cediscor, $\mathrm{n}^{\circ} 4$.

COSNIER, J., (1987), « L'éthologie du dialogue », dans Cosnier J. \& Kerbrat Orecchioni C., Décrire la conversation, Lyon, PUF de Lyon.

COURTILLON, J., (1972), « Quelques aspects de l'accès au sens dans la classe audiovisuelle », Voix et images du CREDIF, $\mathrm{n}^{\circ} 14, \mathrm{p} .1-6$.

DE ANGELIS, G., (2007), Third or additional language acquisition, Clevedon, Multilingual Matters.

DEJEAN-THIRCUIR, Ch. \& MANGENOT, F., (coord.), (2006), Les échanges en ligne dans l'apprentissage et la formation, Le Français dans le Monde, Recherches et Applications, ${ }^{\circ} 40$ (numéro spécial).

DE PIETRO, J.-F., MATTHEY, M. \& PY, B., (1989), « Acquisition et contrat didactique : les séquences potentiellement acquisitionnelles de la conversation exolingue », dans Weil, D. \& Fugier, H., Actes du troisième colloque régional de linguistique, Strasbourg, Université des sciences humaines et Université Louis Pasteur.

DÉVELOTTE, C., KERN, R. \& LAMY, M.-N. (dir.), (2011), Décrire la conversation en ligne : le face à face distanciel, Lyon, ENS Éditions, Collection Langages.

DRISSI, S., (2007), « Dynamiser la communication pédagogique en ligne : aspects socioaffectifs de la fonction tutorale », Échanger Pour Apprendre en Ligne, Grenoble, juin 2007, http://epal.ugrenoble3.fr/dossier/06_act/actes2007.htm

FUCHS, C., (1982), La paraphrase, Paris, PUF.

GALISSON, R., (1979), « Compétence communicative et acquisition des vocabulaires », Travaux de didactique du français langue étrangère, $\mathrm{n}^{\circ} 3$, Université Paul Valéry, Montpellier 3, p.1-33.

GAUDIN, C. \& FLANDIN, S., (2014), « La vidéoformation dans tous ses états : quelles options théoriques, quels scénarios ? Pour quels effets ? Présentation croisée d'un état de l'art », Conférence de consensus, Chaire UNESCO, ENS de Lyon - IFÉ. 
GUICHON, N., (2011), « Former les futurs enseignants de langue en ligne par le biais de la rétrospection », Alsic, Vol. 14, $\mathrm{n}^{\circ} 1$.

GUICHON, N. \& DRISSI, S., (2008), « Tutorat de langue par visioconférence : comment former aux régulations pédagogiques? ", Les Cahiers de l'ACEDLE, Vol. $5, \mathrm{n}^{\circ} 1$.

GÜLICH, E. \& KOTSCHI, Th., (1983), « Les marqueurs de la reformulation paraphrastique », Cahiers de linguistique, $\mathrm{n}^{\circ} 5, \mathrm{p} .305-351$.

GÜLICH, E., (1986), « L'organisation conversationnelle des énoncés inachevés et de leur achèvement interactif en "situation de contact" ", DRALV 34-35, Université Paris 8, p.161-182.

GÜLICH, E. \& KOTSCHI, Th., (1987), « Les actes de reformulation dans la consultation la dame de Caluire ", dans Bange P., L'analyse des interactions verbales. La dame de Caluire : une consultation, Berne, Peter Lang, p.15-81.

JEFFERSON, G., (1972), « Side Sequences », dans Sudnow, D., Studies in Social Interaction, New York, Free Press, p.294-338.

KRESS, G. \& VAN LEEUWEN, Th., (2001), Multimodal Discourse: The Modes and Media of Contemporary Communication, Londres : Arnold.

KRESS, G., (2004), « Reading Images : Multimodality, Representation and New Media », Information Design Journal \& Document Design, 12, n², p.110-119.

LANCIEN, T., (dir.), (2000), Multimédia : les mutations du texte, Cahiers du français contemporain, Lyon, ENS éditions.

LE BOT, M.C., SCHUWER, M. \& RICHARD, E. (dir.), (2008), La reformulation : marqueurs linguistiques, stratégies énonciatives, Rennes, PUR.

LÜDI, G., (1986), « Les marques transcodiques : regards nouveaux sur le bilinguisme », dans Lüdi, G., Devenir bilingue - parler bilingue, Tübingen, Niemeyer, p.1-21.

MARQUILLÓ LARRUY, M. (2012), « Littératie et multimodalité ici \& là-bas... en réponse à Diane Dagenais ", Les cahiers de l'Acedle, vol. $9, n^{\circ} 2$, Notions en Questions en didactique des langues - Les Littératies, p.47-84.

MONDADA, L. (2005), « La construction de l'origo déictique comme travail interactionnel des participants : une approche praxéologique de la spatialité », Intellectica, 2005/2-3, 4142, p.75-100.

OVIATT, Sh. (1999), « Ten myths of multimodal interaction », Communications of the ACM, vol. $42, \mathrm{n}^{\circ} 11, \mathrm{p} .74-81$.

RABATEL, A. (dir.), (2010), Les reformulations pluri-sémiotiques en contexte de formation, Besançon, Presses universitaires de Franche-Comté.

RAST, R. \& TRÉVISIOL, P., (dir.), (2006), L'acquisition d'une langue 3, Acquisition et Interaction en Langue Etrangère, $\mathrm{n}^{\circ} 24$.

ROULET, E., (1987), « Complétude interactive et connecteurs reformulatifs », Cahiers de linguistique française, $\mathrm{n}^{\circ} 8, \mathrm{p} .111-140$.

TELLIER, M. \& STAM, G., (2012), « Stratégies verbales et gestuelles dans l'explication lexicale d'un verbe d'action », dans Rivière, V., Spécificités et diversité des interactions didactiques, Paris, Riveneuve, p.357-374.

VASSEUR, M.-Th., (1991), « Solliciter n'est pas apprendre : initiative, sollicitation et acquisition d'une langue étrangère ", dans Russier, C., Stoffel, H. \& Véronique, D., Interactions en langue étrangère, Aix-en-Provence, Publications de l'Université de Provence, p.49-59. 


\section{ANNEXES}

\section{Conventions de transcription}

Pas de majuscules sauf noms propres (à l'initiale) et syllabes accentuées

3 premières lettres des prénoms des interactants en début de tour de parole (ex:

COR pour Corinne)

MAJ accentuation (ex : BONjour)

: allongement d'une syllabe (ex : je:)

' élision (ex : j'veux)

+ pause brève $(1 \mathrm{sec})$

++ pause moyenne $(2 \mathrm{sec})$

+++ pause longue ( 3 sec et plus)

/ interruption (par un autre locuteur)

- troncation (ex : je retr- retrouve)

[...] chevauchements (ex : COR : c'est pas utile [ça

SAR : ben] pourquoi)

$\uparrow$ intonation montante, montée intonative

$\downarrow$ intonation descendante, chute intonative (ex : tu y vas $\uparrow$ toi $\downarrow$ )

xxx passage inaudible

(?) mot douteux, mot incertain ou meilleur choix

*...* alternance codique (ex : *porque*)

\%A action à valeur de tour de parole (ex : \%A: COR tape une adresse de site)

(...) éléments paraverbaux (ex : rires, soupirs, raclement de gorge, ...)

\%G gestuelle (ex : \%G: pointage, pouce levé, ...)

\%M mimique faciale (ex : \%M: sourire, froncement des sourcils, hochement de tête, écarquillement des yeux, ...)

\%C commentaires

\section{NOTES}

1. Voir le site du français en (première) ligne : http://fle-1-ligne.u-grenoble3.fr

2. Ce cours a été placé sous la responsabilité de Martine Marquillo-Larruy de 2011 à 2013, et sous celle de Pascale Trévisiol-Okamura depuis septembre 2013.

3. Cette contribution reprend pour partie (cf. la dernière analyse: «ça fouette!») une présentation faite par tous les membres de l'équipe (Martine Marquilló Larruy, Pascale TrévisiolOkamura, Christophe Naud et Laure Chotel) dans le cadre d'un symposium au colloque de l'ACEDLÉ à Nantes en juin 2012. À cette occasion Christine Develotte avait apporté son point de vue comme répondante.

4. Dans ce que l'on a appelé les moments de la classe de langue (présentation, explication, répétition - mémorisation, exploitation), la deuxième étape du déroulement de la leçon, l'explication, consiste à faire comprendre. Dans les cinq démarches inventoriées à cette fin, la troisième (partir des acquis des étudiants) propose explicitement de « reformuler ce que [l'on vient] de dire avec d'autres mots» (Besse 1985 : 71). 
5. Dans le $n^{\circ} 6$ des Cahiers du français contemporain, qu'il coordonnait, Thierry Lancien établissait une distinction entre "multicanalité" lorsque l'accent était mis sur les supports et "multimodalité" lorsque l'accent était mis sur leur réception.

6. Pour accéder aux enregistrements vidéo correspondant aux analyses, cliquer ici.

\section{RÉSUMÉS}

Cette étude cherche à décrire le répertoire des stratégies reformulatives et explicatives adoptées par les participants dans le cadre d'un cours de FLE en visioconférence poste à poste. Les données recueillies lors des échanges entre tuteurs francophones et apprenants allophones permettent de mettre en lumière la variété des moyens (verbaux, paraverbaux, mimo-gestuels) mis en oeuvre pour résoudre les problèmes d'intercompréhension ainsi que la complexité des interactions multimodales. Les trois séquences conversationnelles qui sont analysées montrent la façon dont les tuteurs adaptent leurs régulations pédagogiques aux contraintes du dispositif, ce qui peut se traduire par l'usage du clavardage et le recours à une plus grande expressivité mimo-gestuelle pour paraphraser le dit.

This study aims to describe the diversity of reformulative and explanatory strategies adopted by the participants in a French course implying the use of peer to peer video conferencing. The data collected during the exchanges between French tutors and allophone learners allow us to highlight the variety of means (verbal, paraverbal, mimo-gestural) used to solve problems of mutual understanding, as well as the complexity of multimodal interactions. The three conversational sequences analyzed show how the tutors adapt their teaching regulations to the constraints of the device, which may result in the use of live chat and a more expressive body language to paraphrase what has been said.

\section{INDEX}

Keywords : Reformulation, paraphrase, multimodal interactions, teaching regulations, on-line tutoring, synchronous mode.

Mots-clés : Reformulation, paraphrase, interactions multimodales, régulations pédagogiques, tutorat en ligne, mode synchrone.

\section{AUTEURS}

\section{PASCALE TRÉVISIOL-OKAMURA}

Université Paris 3 Sorbonne Nouvelle - DILTEC, EA 2288

MARTINE MARQUILLÓ-LARRUY

Université Lyon II, Adis-langues, ICAR - UMR 5191 